\title{
Yttrium-90 radioembolization in the management of liver tumors: expanding the global experience
}

\author{
Ahsun Riaz $\cdot$ Riad Salem
}

Published online: 24 December 2009

(C) Springer-Verlag 2009

\begin{abstract}
Although liver metastases are more common than primary liver malignancies, the incidence of hepatocellular carcinoma (HCC) continues to rise [1]. Surgical and medical therapies play a limited role in the management of advanced hepatic malignancies [2-4]. Locoregional therapies include ablative (e.g. radiofrequency ablation) and transarterial (e.g. chemoembolization) procedures. The unique anatomy of the liver and its tumors (relative hyperperfusion of liver tumors via the hepatic artery) allows targeted delivery of transarterial therapeutic agents.

Historically, radiation therapy has played a limited role in the treatment of liver tumors primarily because of the low hepatic tolerance to external beam radiation [5]. Radiation-induced liver disease, a syndrome of anicteric hepatomegaly, ascites and elevated liver enzymes, is one of the most important treatment-related complications of radiation therapy [6]. However, newer technologies associated with external beam radiation have increased the safety profile. Radioembolization is a transarterial locoregional therapy for liver tumors, with numerous radionuclide/carrier combinations having been studied worldwide.
\end{abstract}

This Editorial Commentary refers to the article http://dx.doi.org/ 10.1007/s00259-009-1279-6

A. Riaz $\cdot$ R. Salem

Department of Radiology, Section of Interventional Radiology,

Northwestern Memorial Hospital,

Robert H. Lurie Comprehensive Cancer Center,

Chicago, IL, USA

R. Salem $(\bowtie)$

Section of Interventional Radiology, Department of Radiology, Northwestern University Feinberg School of Medicine,

676 North St. Clair Street, Suite 800,

Chicago, IL 60611, USA

e-mail: r-salem@northwestern.edu
There are two formulations of ${ }^{90} \mathrm{Y}$ microspheres being used. TheraSphere (MDS Nordion, Ottawa, Canada) is composed of 15-30 $\mu \mathrm{m}$ nonbiodegradable glass microspheres with ${ }^{90} \mathrm{Y}$ as an integral constituent of the glass. It has been FDA-approved for use in HCC patients with or without portal vein thrombosis. SIR-Spheres (SIRTex Medical, Lane Cove, Australia) are composed of resin microspheres and have been FDA-approved for use in metastatic colorectal carcinoma. Both agents have regulatory approval for liver neoplasia in Europe and various countries worldwide.

Our group recently published an analysis of 291 patients focusing on the outcomes following radioembolization [7]. We concluded that this treatment was safe and effective in patients with HCC. Patients with coexisting portal vein invasion and poor liver function (Child-Pugh B or C) had poor outcomes. This treatment was shown to be safe if proper precautions and meticulous technique are applied [8].

In this first European experience with glass microspheres, Garin et al. should be commended for thoroughly investigating and providing proof of concept for the use of ${ }^{90} \mathrm{Y}$ in liver malignancies [9]. Although in our experience angiography and planar SPECT has been sufficient to provide safe treatment, can SPECT/CT further increase the safety profile of this treatment option? Can it provide information on dosimetry? The authors present an interesting concept involving the utilization of SPECT/CT prior to and after administration of ${ }^{90} \mathrm{Y}$. It will be interesting to see data from larger cohorts on the role of this technique and its ability to reduce complications following administration of ${ }^{90} \mathrm{Y}$. However, we should be cautious in not necessarily supporting the use of this approach, as we have demonstrated in our center that ${ }^{90} \mathrm{Y}$ can be given safely without SPECT/ CT. Further refinement of the role of SPECT/CT is required. Garin et al. also present a case of a dramatic effect (imaging 
response, alpha-fetoprotein decrease) following radioembolization. Such dramatic effects have also been seen in other centers. The roles of biomarkers such as alpha-fetoprotein in response assessment following HCC continues to evolve [10].

Garin et al. correctly point out that there are technical aspects that require attention with this therapy [9]. As with all complex therapies (surgical resection, liver transplantation), there is a learning curve for the team involved in administering the treatment. Similar to complex surgical therapies, this approach also requires dedicated and focused centers with a multidisciplinary team able to offer systemic, surgical and locoregional therapies for liver malignancies. It has been universally noted in other centers that once the team is beyond the learning curve, the procedure with this technique becomes routine and straightforward. Furthermore, the specific advantages of this therapy over other more complex embolization procedures include the fact that it is performed on an outpatient basis and that fewer treatments are required. These differences are not to be ignored; therapies that can be administered with a fewer treatment sessions and optimal patient comfort clearly have an impact on quality of life. This is relevant since most of these options are palliative.

We believe that there is still work to be done. The dosimetry for TheraSphere is based on the Medical Internal Radiation Dose model, and SIR-Sphere uses the partition, the empirical or the body surface area model. Thorough dose escalation and dose fractionation studies need to be performed. The low incidence of radiation-induced liver disease being reported brings into question whether the optimum tumoricidal dose has been reached using the current dosimetry models. Hence in certain settings, it is possible that even higher doses of radiation may be delivered using radioactive microspheres. An objective standardized method for calculating relative tumor hypervascularity will also assist in formulating a better dosimetry model, since most metastatic lesions to the liver (with the exception of neuroendocrine tumors) are not as hypervascular as HCC.

Garin et al. raise two important points which could be the focus of future analyses [9]. It is necessary to explore the role of radioembolization in combination with other therapies such as systemic chemotherapeutic agents, biological agents and radiosensitizing agents. Studies are required to understand the effects of the optimum combination of these therapies with radioembolization. The "chemoradiation" paradigm, standard in radiation oncology, needs to be expanded to liver tumors. This technology permits this concept to be explored. Also, econometric analyses comparing the costs of radioembolization (an outpatient procedure) to the costs of other therapies are also required. We completely agree with the investigators.

We look forward to more data from Dr. Garin and his colleagues as well as others in Europe and worldwide that continue to build and validate the body of evidence of radioembolization in treating liver tumors.

Conflicts of interest R.S. is an advisor to MDS Nordion. None of the authors has disclosed a conflict of interest.

\section{References}

1. Parkin DM, Bray F, Ferlay J, Pisani P. Global cancer statistics, 2002. CA Cancer J Clin 2005;55:74-108.

2. Llovet JM, Ricci S, Mazzaferro V, Hilgard P, Gane E, Blanc JF, et al. Sorafenib in advanced hepatocellular carcinoma. N Engl J Med 2008;359:378-90.

3. Mazzaferro V, Regalia E, Doci R, Andreola S, Pulvirenti A, Bozzetti F, et al. Liver transplantation for the treatment of small hepatocellular carcinomas in patients with cirrhosis. N Engl J Med 1996;334:693-9.

4. Carpizo DR, D'Angelica M. Liver resection for metastatic colorectal cancer in the presence of extrahepatic disease. Lancet Oncol 2009;10:801-9.

5. Stillwagon GB, Order SE, Guse C, Klein JL, Leichner PK, Leibel SA, et al. 194 hepatocellular cancers treated by radiation and chemotherapy combinations: toxicity and response: a Radiation Therapy Oncology Group Study. Int J Radiat Oncol Biol Phys 1989;17:1223-9.

6. Dawson LA, Normolle D, Balter JM, McGinn CJ, Lawrence TS, Ten Haken RK. Analysis of radiation-induced liver disease using the Lyman NTCP model. Int J Radiat Oncol Biol Phys 2002;53:810-21.

7. Salem R, Lewandowski RJ, Mulcahy MF, Riaz A, Ryu RK, Ibrahim $\mathrm{S}$, et al. Radioembolization for hepatocellular carcinoma using Yttrium-90 microspheres: a comprehensive report of long-term outcomes. Gastroenterology. 2009.

8. Riaz A, Lewandowski RJ, Kulik LM, Mulcahy MF, Sato KT, Ryu $\mathrm{RK}$, et al. Complications following radioembolization with yttrium-90 microspheres: a comprehensive literature review. J Vasc Interv Radiol. 2009;20:1121-30. quiz 31.

9. Garin E, Rolland Y, Boucher E, Ardisson V, Laffont S, Boudjema $\mathrm{K}$, et al. First experience of hepatic radioembolization using microspheres labelled with yttrium-90 (TheraSphere): practical aspects concerning its implementation. Eur J Nucl Med Mol Imaging. 2009. doi:10.1007/s00259-009-1279-6.

10. Riaz A, Ryu RK, Kulik LM, Mulcahy MF, Lewandowski RJ, Minocha $J$, et al. Alpha-fetoprotein response after locoregional therapy for hepatocellular carcinoma: oncologic marker of radiologic response, progression, and survival. J Clin Oncol 2009;27:5734-42. 